Commun. Fac. Sci. Univ. Ank. Series Al

V. 45. pp. 45-50 (1996)

\title{
ON HELICES OF A LORENTZIAN MANIFOLD
}

N. EKMEKÇİ and H.H. HACISALIHHOĞLU

Department of Mathematics, Ankara University, Ankara, TURKEY

(Received Sep. 29, 1995; Revised March 7, 1996: Accepted March 19, 1996)

\section{ABSTRACT}

T. Ikawa obtained in [1] the following differential equation

$$
\mathrm{D}_{\mathrm{x}} \mathrm{D}_{\mathrm{x}} \mathrm{D}_{\mathrm{x}} \mathrm{X}-\mathrm{KD}_{\mathrm{x}} \mathrm{X}=0, \mathrm{~K}=\mathrm{k}_{1}^{2}-\mathrm{k}_{2}^{2}
$$

for the circular helix which corresponds to the case that the curvatures $k_{1}$ and $k_{2}$ of $a$ time-like curve $\alpha$ on the Lorentzian manifold $M_{1}$ are constants.

In this paper, $T$. lkawa's result is generalized to the case of general helix, i.e. $k_{1}$ and $k_{2}$ are non-constant functions of $t$, but $\frac{k_{1}}{k_{2}}$ is constant.

\section{PRELIMINARIES}

$\mathbb{R}^{\mathrm{n}}$ with the metric tensor

$$
\left\langle\mathrm{V}_{\mathrm{p}}, \mathrm{W}_{\mathrm{p}}\right\rangle=-\sum_{\mathrm{j}=1}^{\mathrm{i}} \mathrm{V}_{\mathrm{j}} \mathrm{W}_{\mathrm{j}}+\sum_{\mathrm{k}=\mathrm{i}+1}^{\mathrm{n}} \mathrm{V}_{\mathrm{k}} \mathrm{W}_{\mathrm{k}}, \mathrm{V}_{\mathrm{p}}, \mathrm{W}_{\mathrm{p}} \in \mathbb{R}^{\mathrm{n}}
$$

is called semi-Euclidean space and is denoted by $R_{i}^{n}$ where $i$ is called the index of the metric [2].

Let $M$ be an n-dimensional smooth manifold equipped with a metric $\langle$,$\rangle which is a symmetric non-degenerate (0,2)$-tensor field on $M$ with constant index.

A tangent space $T_{p}(M)$ at the point $p \in M$ is furnished with the canonical inner product. If the index of the metric $\langle$,$\rangle is i$, then we call $M$ and indefinite-Riemannian manifold of index $i$ and denote it by $M_{i}$. If $\langle$,$\rangle is positive definite, then M$ is a Riemannian manifold. Especially if $\mathrm{i}=1$, then $M$ is called a Lorentzian manifold. A tangent vector $X$ of $M_{i}$ is said to be space like if $\langle X, X\rangle>0$, time-like if $\langle X, X\rangle\langle 0$ and null if $\langle X, X\rangle=0$ and $X \neq 0$. 
Let $X_{1}, \ldots, X_{i}, X_{i+1}, \ldots, X_{n}$ be tanget vectors of $M_{i}, n=\operatorname{dim} M$. Assume that they satisfy $\left\langle X_{A}, X_{B}\right\rangle=\varepsilon_{A} \delta_{A B}$ where $\varepsilon_{A}=\left\langle X_{A}, X_{A}\right\rangle=+1$ (resp. -1) for $A=1,2, \ldots, n$. If each $X_{A}$ is space-like (resp. time-like) then $\left\{X_{1}, \ldots, X_{n}\right\}$ is called an orthonormal basis of $M_{i}$ [2].

\section{CURVES}

A curve in an indefinite-Riemannian manifold $M_{i}$ is a smooth mapping

$$
\alpha: I \rightarrow M_{i}
$$

where $I$ is an open interval in the real line $\mathbb{R}$. The interval $I$ has a coordinate system consisting of the identity map $u$ of $I$. The velocity vector of $\alpha$ at $t \in I$ is

$$
\alpha^{\prime}(\mathrm{t})=\frac{\mathrm{d} \alpha(\mathrm{u})}{\mathrm{du}}
$$

A curve $\alpha$ is said to be regular if $\alpha^{\prime}(t)$ does not vanish for all $t$ in I.

A curve $\alpha$ in an indefinite-Riemannian manifold $M_{i}$ is said to be space-like if its velocity vectors $\alpha^{\prime}$ are space-like for all $t \in I$; similarly for time-like and null. If $\alpha$ is a space-like or time-like curve, we can reparametrize it such that $\left\langle\alpha^{\prime}(t), \alpha^{\prime}(t)\right\rangle=\varepsilon$ (where $\varepsilon=+1$ if $\alpha$ is space-like and $\varepsilon=-1$ if $\alpha$ is time-like respectively). In this case $\alpha$ is said to be unit speed or it has arc length parametrization. Here and in the sequal, we assume that the space-like or time-like curve $\alpha$ has an arc length parametrization.

Wc define here a circle and circular helix in an indefinite-Riemannian manifold $M_{i}$ (cf[3], [4]). Let $\alpha$ be a time-like curve in $M_{i}$. By $k_{j}$, we denote the $\mathrm{j}$-th curvature of $\alpha$. If $\mathrm{k}_{\mathrm{j}}$ vanishes for $\mathrm{j}>2$ an the principal vector field $\mathrm{Y}$ and binormal vector field $\mathrm{Z}$ are space-like, then we have the following Frenet formulas along $\alpha$

$$
\begin{aligned}
\alpha^{\prime}(t) & =: X \\
D_{x} X & =k_{1} Y \\
D_{x} Y & =k_{1} X+k_{2} Z \\
D_{x} Z & =-k_{2} Y
\end{aligned}
$$

where $D$ denotes the covariant differentiation in $M_{i}$. A curve $\alpha$ is called a circle if $k_{2} \equiv 0$ and $k_{1}=$ constant $>0$, for all $t \in I$. 
If both $k_{1}$ and $k_{2}$ are positive constants along $\alpha$, then $\alpha$ is called a circular helix [1].

Definition 2.1: A general helix is a regular curve $\alpha$ such that for some fixed unit vector $U,\langle T, U\rangle$ is constant. $U$ is called the axis of a helix [5].

Corollary: (Lancert, 1802). A unit speed curve $\alpha$ with $k_{2} \neq 0$ is a general helix if and only if there is a constant c such that $k_{1}(t)=c_{2}(t)$ for all $t \in I$ [5].

\section{CIRCLES}

Let $\alpha$ be a regular time-like curve in a Lorentzian manifold $M_{1}$. In this section, we assume that $\alpha$ is a circle, that is, $\alpha$ satisfies

$$
\left.\begin{array}{rl}
\alpha^{\prime}(t) & =X \\
D_{x} X & =k_{1}(t) Y \\
D_{x} Y & =k_{1}(t) X
\end{array}\right\}
$$

for any $t \in I$, where $Y$ is a space-like vector field and $k_{1}$ a positive constant function of the parameter $t$.

Lemma 3.1: Let $\alpha$ be a time-like curve in a Lorentzian manifold $M_{1}$. If $\alpha$ is a circle, then the velocity vector field $X$ or $\alpha$ satisfies

$$
\mathrm{D}_{\mathrm{X}} \mathrm{D}_{\mathrm{X}} \mathrm{X}-\left\langle\mathrm{D}_{\mathrm{X}} \mathrm{X}, \mathrm{D}_{\mathrm{X}} \mathrm{X}\right\rangle \mathrm{X}=0
$$

conversely, if the velocity vector field of a time-like curve $\alpha$ satisfies (3.2), then $\alpha$ is either a geodesic or a circle [1].

\section{HELICES}

Next we consider general helices in a Lorentzian manifold $\mathbf{M}_{1}$. Then we have

$$
\left.\begin{array}{rl}
\alpha^{\prime}(t) & =X \\
D_{x} X & =k_{1} Y \\
D_{x} Y & =k_{1} X+k_{2} Z \\
D_{x} Z & =-k_{2} Y
\end{array}\right\}
$$

for any $t \in I$, where $Y, Z$ are space-like vector fields and $k_{1}, k_{2}$ are the functions of the parameter $t$. 
Theorem 4.1: A unit speed curve $\alpha$ on $M_{1}$ is a general helix if and only if

$$
D_{x} D_{x} D_{x} X-\bar{K} D_{x} X=3 k_{1}^{\prime}(t) D_{x} Y
$$

where

$$
\overline{\mathrm{K}}=\frac{\mathrm{k}_{1}^{\prime \prime}(\mathrm{t})}{\mathrm{k}_{1}^{\prime}(\mathrm{t})}+\mathrm{k}_{1}^{2}(\mathrm{t})-\mathrm{k}_{2}^{2}(\mathrm{t})
$$

Proof: Suppose that $\alpha$ is a general helix. Then, from (4.1), we have,

$$
\begin{aligned}
D_{x} D_{x} X & =D_{x}\left(k_{1} Y\right) \\
& =k_{1}^{\prime} Y+k_{1} D_{x} Y \\
& =k_{1}^{2} X+k_{1}^{\prime} Y+k_{1} k_{2} Z
\end{aligned}
$$

and

$$
\begin{aligned}
D_{x} D_{x} D_{x} X & =3 k_{1}^{\prime} k_{1} X+\left(k_{1}^{\prime \prime}-k_{1} k_{2}^{2}\right) Y \\
& +\left(k_{1}^{\prime} k_{2}+\left(k_{1} k_{2}\right)^{\prime}\right) Z+k_{1}^{2} D_{x} X
\end{aligned}
$$

Now, since $\alpha$ is a general helix, we have

$$
\frac{k_{1}}{k_{2}}=\text { constant }
$$

and this upon the derivation gives rise to

$$
k_{1}^{\prime} k_{2}=k_{1} k_{2}^{\prime} \text {. }
$$

If we substitute the values

$$
Y=\frac{1}{k_{1}(t)} D_{X} X
$$

and

$$
\left(k_{1}(t) k_{2}(t)\right)^{\prime}=2 k_{1}^{\prime}(t) k_{2}(t),
$$

in (4.5) we obtain

$$
\begin{aligned}
D_{x} D_{x} D_{x} X & =\left(\frac{k_{1}^{\prime \prime}(t)}{k_{1}(t)}+k_{1}^{2}(t)-k_{2}^{2}(t)\right) D_{x} X+3 k_{1}^{\prime}(t)\left(k_{1}(t) X+k_{2}(t) Z\right) \\
& =\left(\frac{k_{1}^{\prime \prime}(t)}{k_{1}(t)}+k_{1}^{2}(t)-k_{2}^{2}(t)\right) D_{x} X+3 k_{1}^{\prime}(t) D_{x} Y .
\end{aligned}
$$


Hence we have (4.2).

Conversely let us assume that (4.2) holds. We show that the curve $\alpha$ is a general helix. Differentiating covariantly (4.6) we obtain

and so,

$$
D_{x} Y=-\frac{k_{1}^{\prime}(t)}{k_{1}^{2}(t)} D_{x} X+\frac{1}{k_{1}(t)} D_{x} D_{x} X
$$

$$
\begin{gathered}
D_{x} D_{x} Y=\left(-\frac{k_{1}^{\prime}(t)}{k_{1}^{2}(t)}\right)^{\prime} D_{x} X-\frac{k_{1}^{\prime}(t)}{k_{1}^{2}(t)} D_{x} D_{x} X \\
-\frac{k_{1}^{\prime}(t)}{k_{1}^{2}(t)} D_{x} D_{x} X+\frac{1}{k_{1}(t)} D_{x} D_{x} D_{x} X
\end{gathered}
$$

if we use (4.2) in (4.7), we get

$$
\begin{gathered}
D_{x} D_{x} Y=\left\{\left(-\frac{k_{1}^{\prime}(t)}{k_{1}^{2}(t)}\right)^{\prime}+\frac{\bar{K}}{k_{1}(t)}\right\} D_{x} X \\
-\frac{2 k_{1}^{\prime}(t)}{k_{1}^{2}(t)} D_{x} D_{x} X+\frac{3 k_{1}^{\prime}(t)}{k_{1}(t)} D_{x} Y .
\end{gathered}
$$

Substituting (4.4) and (4.1) in this last equality we obtain

$$
\begin{aligned}
& D_{x} D_{x} Y=\left\{\left(-\frac{k_{1}^{\prime}(t)}{k_{1}^{2}(t)}\right)^{\prime}+\frac{\bar{K}}{k_{1}(t)}\right)_{x} D_{x} \\
& -\frac{2 k_{1}^{\prime 2}(t)}{k_{1}^{2}(t)} Y+k_{1}^{\prime}(t) X+\frac{k_{1}^{\prime}(t) k_{2}(t)}{k_{1}(t)} Z
\end{aligned}
$$

On the other hand substituting the equality

$$
D_{x} D_{x} Y=k_{1}^{\prime}(t) X-k_{2}^{2}(t) Y+k_{2}^{\prime}(t) Z+k_{1}(t) D_{x} X
$$

in $(4.8)$ we obtain

and so

$$
k_{2}^{\prime}(t)=\frac{k_{1}^{\prime}(t) k_{2}(t)}{k_{1}(t)}
$$

$$
\frac{k_{2}^{\prime}(t)}{k_{2}(t)}=\frac{k_{1}^{\prime}(t)}{k_{1}(t)}
$$


Integrating this we get

$$
\frac{k_{1}(t)}{k_{2}(t)}=\text { constant }
$$

Thus $\alpha$ is a general helix. Hence the proof is done.

We note that in the special case when $\alpha$ is a circular helix, our theorem coincides with the result of $T$. Ikawa [1].

\section{REFERENCES}

[1] IKAWA, T., On Curves and Submanifolds in an Indefinite-Riemannian Manifold, Tsukuba J. Math. 9 (1985), 353-371,

[2] O'NEIL, B., Semi-Rienannian Geometry, Academic Press, New York, 1983.

[3] BONNER, W.B., Null Curves in a Minkowski Space-Time Tensor N.S., 20 (1969), 229-242.

[4] NOMIZU, K., YANO, K., On Circles and Spheres in Riemannian Geometry, Math. Ann., 210 (1974), 163-170.

[5] MiLlmaN, R.S., PARKER, G.D., Elements of Differential Geometry, Prentice Hall, Englewood Cliffs, New Jersey, 1987. 